\title{
Nuevos casos de feocromocitoma en el hospital universitario de Neiva
}

\author{
Alejandro Pinzón Tovar ${ }^{1}$,Eduard Mauricio Díaz ${ }^{2}$, Orfa Yaneth Motta ${ }^{3}$ Marcela Castro ${ }^{4}$
}

${ }^{1}$ Médico Internista, Universidad Surcolombiana, Neiva.

Endocrinólogo Universidad Militar Nueva Granada. Médico

Internista y Endocrinólogo del Hospital Universitario Hernando

Moncaleano Perdomo Neiva.

${ }^{2,3,4}$ Médico residente de III año Medicina Interna, Universidad

Surcolombiana Neiva.

Correspondencia: Alejandro Pinzón Tovar. alepyto@yahoo.com

\section{Resumen}

$\mathrm{E}$ I feocromocitoma es un tumor productor de catecolaminas con una prevalencia de 2 a 8 por millón de personas, que deriva en $85 \%$ de los casos de las células cromafines de la médula suprarrenal. La presente revisión, ilustrada con una serie de casos, brinda una actualización del tema que muestra situaciones de la vida real que ocurren en nuestra institución. Las dificultades para el estudio y diagnóstico, por la pobre disponibilidad de las pruebas para determinar el exceso de catecolaminas o sus metabolitos y lo infrecuente de la condición, pueden retardar la sospecha de esta patología como una posibilidad diagnóstica y diferir la interconsulta a endocrinología, necesaria para aportar en el tratamiento de los casos de feocromocitoma. Con este documento describimos lo que ocurre en nuestra población y realizamos una revisión práctica del estudio, diagnóstico y manejo actual de este tipo de tumores.

Palabras claves: Feocromocitoma, incidentaloma adrenal, neurofibromatosis, hipertensión, catecolaminas.

\section{Abstract}

Pheochromocytoma is a catecholamine producing tumor with a prevalence of 2 to 8 per million people, $85 \%$ arise from chromaffin cells of the adrenal medulla. The present review, illustrated with a case series, gives an update on the issue showing real life situations that occur in our institution. The difficulties in the study and diagnosis by the poor availability of tests for excess catecholamines or their metabolites and uncommon condition, can slow the suspicion of this disease as a diagnostic possibility and defer interconsultation to endocrinology, necessary for contribute to treat pheochromocytoma cases. In this paper we describe what happens in our community and realize a practical review in the study, diagnosis and current management of these tumors.

Key words: Pheochromocytoma, adrenal incidentaloma, neurofibromatoses, hypertension, catecholamines.

\section{Introducción}

La primera descripción de un feocromocitoma fue realizada en 1886 por F. Frankel, cuando una joven con ataques episódicos de cefalea, palpitaciones y ansiedad murió repentinamente; la necropsia reveló tumores de médula adrenal bilaterales. El 85\% de los feocromocitomas son benignos y el 15\%, malignos; éstos, generalmente son extradrenales (24\% vs $7 \%$ ) (1).Cerca del $10 \%$ de los feocromocitomas son heredados y un 25\% de los esporádicos sin antecedentes familiares albergan una mutación germinal heredada ${ }^{(2)}$. El objetivo de este artículo es presentar una serie de casos y realizar una revisión de la literatura sobre una patología descrita desde hace más de 100 años, que no es muy común pero que se presenta con una alta morbilidad y mortalidad. La presentación incidental, las dificultades en el estudio y el abordaje inicial por grupos quirúrgicos son temas comunes en nuestro hospital. Debido a la ausencia de publicaciones locales, esperamos que este texto sirva de referencia regional, con recomendaciones acordes con nuestros recursos.

\section{Casos clínicos}

\section{Caso clínico 1}

Adolescente masculino de 15 años, sin antecedentes de importancia, que consulta por dolor abdominal en hipocondrio izquierdo, síntomas generales y fiebre. Manejado como infección de vías urinarias sin mejoría. Se estudia con TAC de abdomen que reporta lesiones sólidas bilaterales dependientes de las adrenales; la mayor, del lado izquierdo de $7 \mathrm{~cm}$ de diámetro mayor. Le realizaron resonancia magnética (RM) (figura 1) y biopsia de masa adrenal izquierda. El estudio histopatológico reporta feocromocitoma. Con este informe se interconsulta a Endocrinología que solicita perfil bioquímico (tabla 1). Los resultados apoyan el diagnóstico de feocromocitoma, el calcio sérico es normal, la ecografía de cuello reporta una lesión nodular de $2 \mathrm{~cm}$, localizada en región submaxilar 
Figura 1. Lesiones sólidas dependientes de las glándulas adrenales. Nótese el mayor tamaño de la masa izquierda. CASO 1

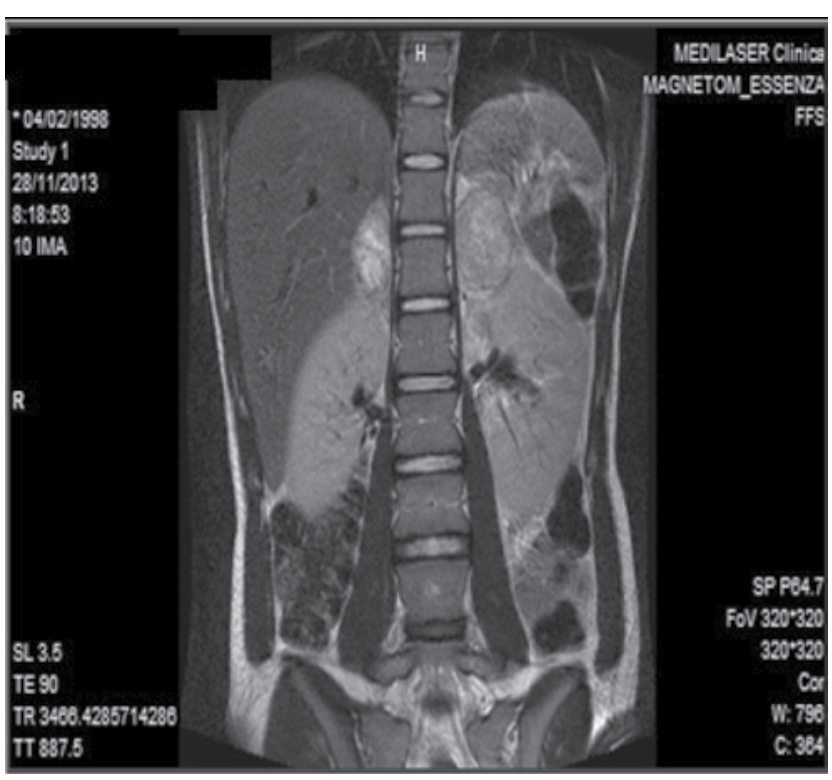

Tabla 1. Estudio bioquímico CASO 1

\begin{tabular}{l|c|c}
\multicolumn{1}{c|}{ Laboratorio } & Resultado & $\begin{array}{c}\text { Valor de } \\
\text { referencia }\end{array}$ \\
\hline Adrenalina en plasma & $430 \mathrm{pg} / \mathrm{ml}$ & $0-100$ \\
\hline Noradrenalina en plasma & $491,3 \mathrm{pg} / \mathrm{ml}$ & $0-600$ \\
\hline Metanefrinas totales orina & $41.239 \mu \mathrm{g} / 24$ horas & Menor de 900 \\
\hline Normetanefrina orina & $15.019 \mu \mathrm{g} / 24$ horas & Menor de 600 \\
\hline Metanefrina orina & $35 \mu \mathrm{g} / 24$ horas & Menor de 300 \\
\hline Cortisol libre en orina & $155,20 \mu \mathrm{g} / 24$ horas & $20-130$ \\
\hline
\end{tabular}

derecha. Se solicita gammagrafía con metayodobencilguanidina que evidencia hipercaptación patológica a nivel adrenal de forma bilateral sin lesiones metastásicas o de tumores sincrónicos (figura 2). Iniciamos alfa bloqueo hasta lograr hipotensión ortostática y luego se adiciona bloqueo beta. En junta médicoquirúrgica se decide llevar a adrenalectomía laparoscópica bilateral. Durante la resección del tumor derecho presenta descarga catecolaminérgica alcanzando tensión arterial media de $183 \mathrm{mmHg}$. La evolución posquirúrgica fue adecuada. La patología describe una masa adrenal derecha de $5 \times 3 \times 2,5 \mathrm{~cm}$ e izquierda de 7 × 4,5 × $4 \mathrm{~cm}$ con múltiples adherencias. Áreas de necrosis confluente y múltiples focos de trombos neoplásicos en vasos e invasión capsular sin extensión al tejido adiposo peri-
Figura 2. Gamagrafía con metayodobencilguanidina

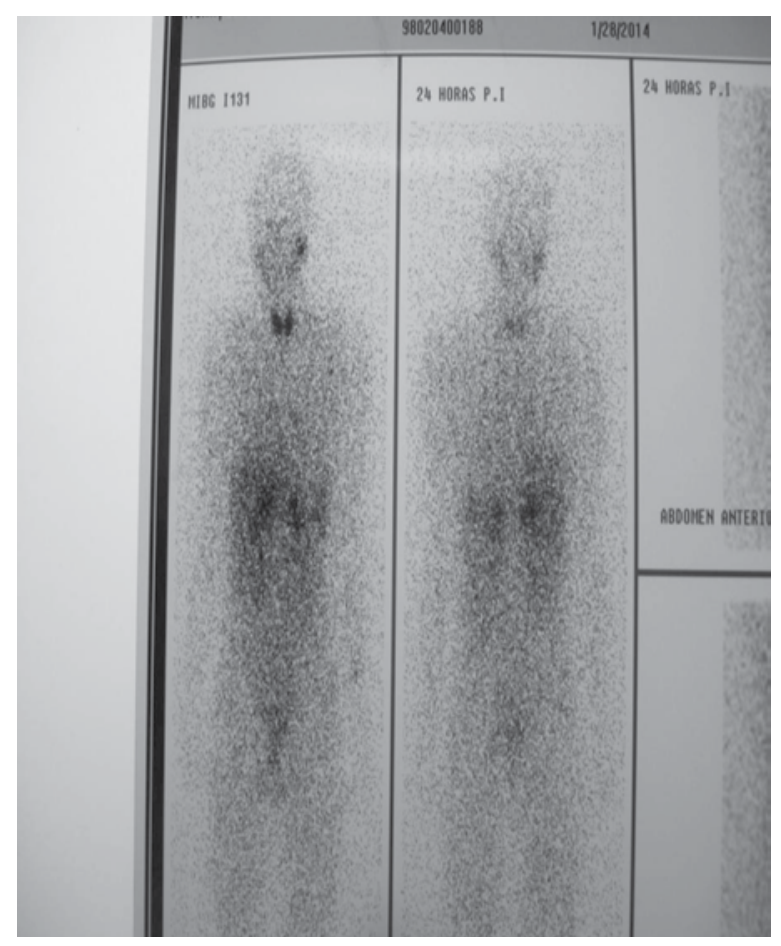

Patrón gamagráfico compatible con la presencia de tumores neuroectodérmicos tipo feocromocitoma de localización suprarrenal y sin evidencia de lesiones metastásicas.

glandular (figura 3). Los hallazgos histológicos corresponden a feocromocitoma bilateral. La inmunohistoquímica fue positiva para cromogranina, Ki67 del 2\% y ausencia en múltiples áreas de células sustentaculares con la marcación S100.

\section{Caso clínico 2}

Paciente masculino de 48 años, procedente del Caquetá, ingresa a urgencias por dolor abdominal tipo cólico localizado en hipocondrio derecho, irradiado a región lumbar con náuseas, hiporexia e historia de pérdida de $7 \mathrm{Kg}$ de peso. En TAC de abdomen se detecta masa hipodensa en polo superior del Figura 3. Masa, suprarrenales derecha e izquierda. CASO 1

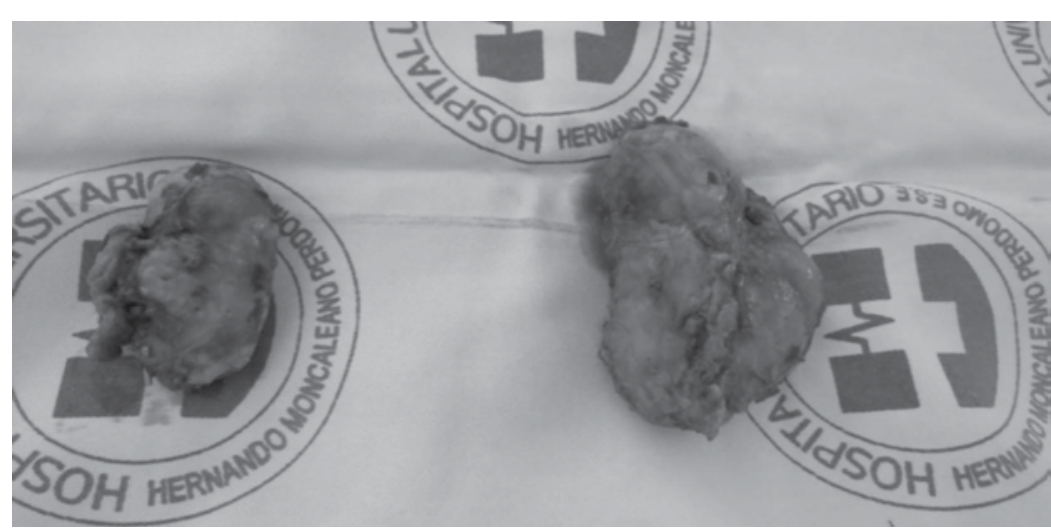


Figura 4. Masa heterogénea en el polo superior del riñón derecho. CASO 2

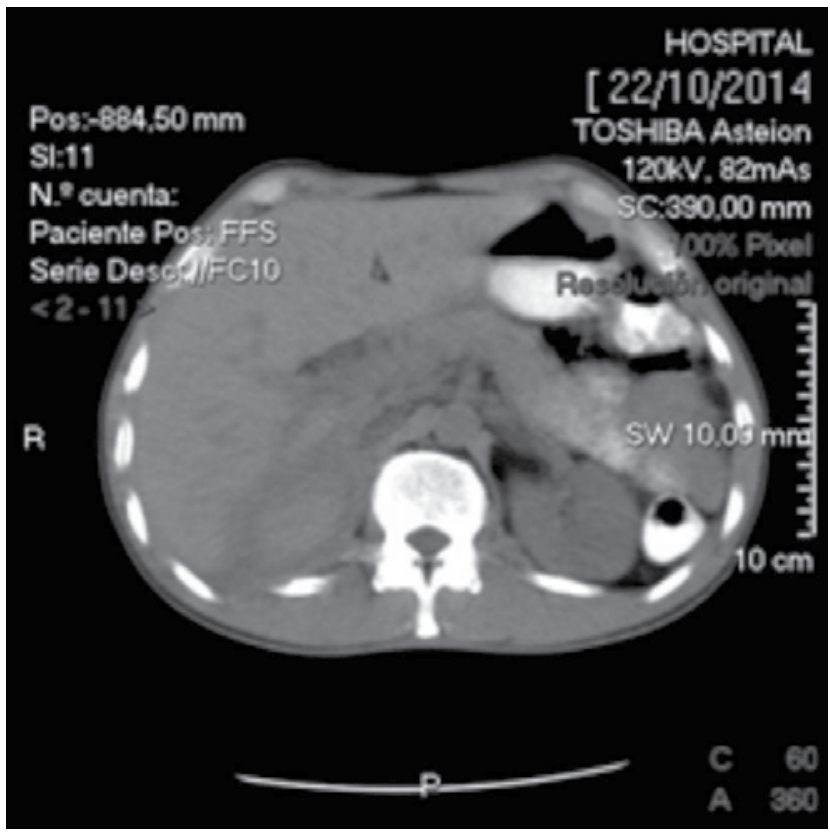

Figura 5. Riñón derecho y tumor adrenal con extensa necrosis isquémica

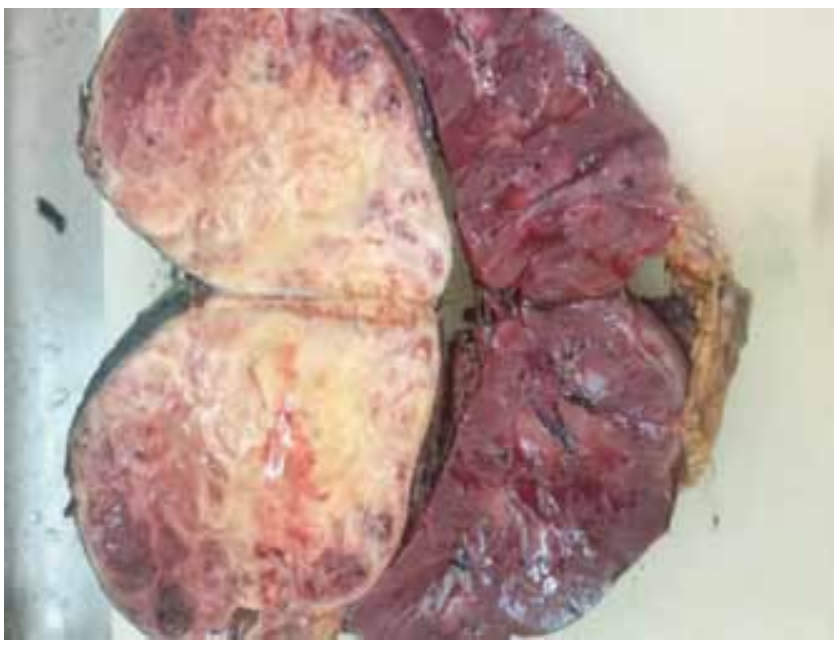

riñón derecho. La lesión es dependiente de la glándula adrenal derecha, de $9 \times 7,5 \mathrm{~cm}$ y muestra discreto realce central y periférico (figura 4). El servicio de Urología realiza resección de masa adrenal, nefrectomía derecha y linfadenectomía retroperitoneal; el informe de patología reporta un tumor adrenal con necrosis isquémica del $80 \%$ (figura 5). La inmunohistoquímica es positiva para cromogranina, sinaptofisina, inhibina, vimentina y S100 con índice de proliferación Ki67 del 10\% y marcación focal para coctel de queratinas AE1/AE3; siendo negativa para Malan-A; CD10 y RCC. Patología considera que es un feocromocitoma.

\section{Caso clínico 3}

Hombre de 62 años, procedente de Neiva, con historia de neurofibromas (figura 6) e hipertensión arterial (HTA), ingresa por emergencia hipertensiva con órgano blanco cerebro. Durante la evolución, su HTA es de difícil control y requiere varios fármacos antihipertensivos a dosis máximas. El servicio tratante solicita tomografía (TAC) de abdomen que muestra una lesión adrenal derecha heterogénea (figura 7). Con este

Figura 6. Lesiones múltiples en tórax compatibles con neurofibromas. CASO 3

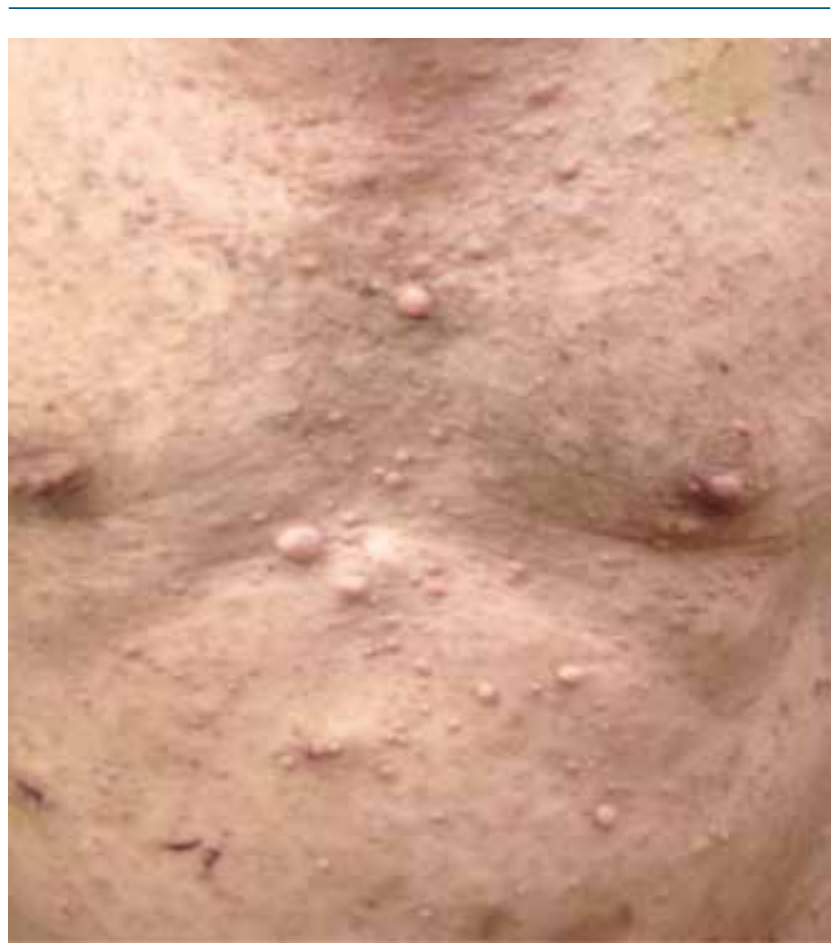

Figura 7. Lesión expansiva suprarrenal derecha. CASO 3.
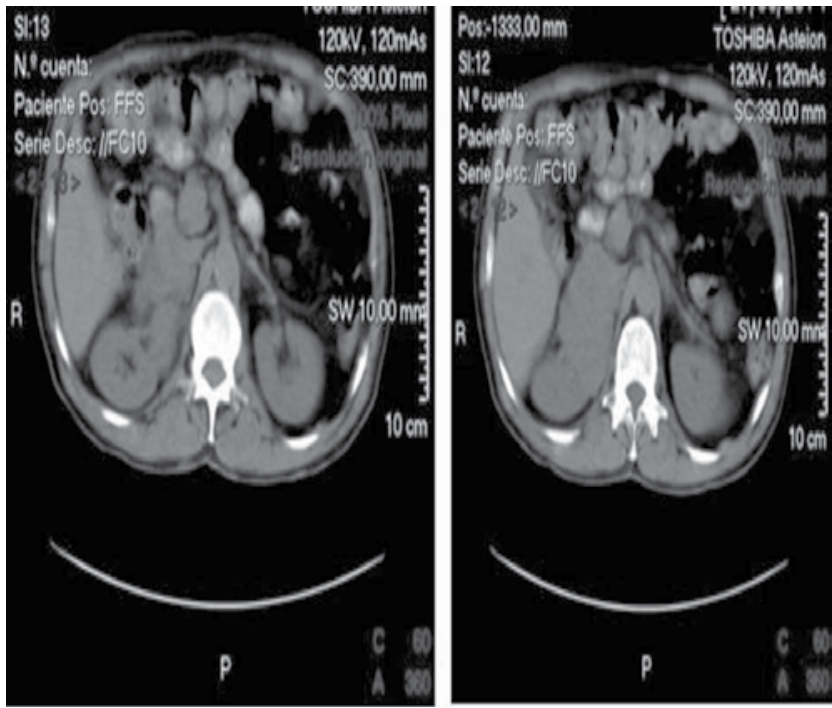

Revista Colombiana de Endocrinología, Diabetes y Metabolismo 
Tabla 2. Estudio bioquímico CASO 2

\begin{tabular}{l|c|c}
\hline \multicolumn{1}{c|}{ Laboratorio } & Resultado & Valor de referencia \\
\hline Adrenalina en plasma & $13,9 \mathrm{pg} / \mathrm{ml}$ & $0-100$ \\
\hline Noradrenalina en plasma & $699,3 \mathrm{pg} / \mathrm{ml}$ & $0-600$ \\
\hline Metanefrinas libres en plasma & $12,1 \mathrm{pg} / \mathrm{ml}$ & Menor de $90 \mathrm{pg} / \mathrm{ml}$ \\
\hline Metanefrinas totales orina & $3678 \mu \mathrm{g} / 24$ horas & Menor de 600 \\
\hline Normetanefrina orina & $3643 \mu \mathrm{g} / 24 \mathrm{horas}$ & Menor de 300 \\
\hline Metanefrina orina & $35 \mu \mathrm{g} / 24 \mathrm{horas}$ & Menor de 600 \\
\hline Metanefrina/creatinina orina & $3034 \mu \mathrm{g} / \mathrm{g} \mathrm{de} \mathrm{creatinina}$ & $0-100$ \\
\hline Cromogranina A & $18,6 \mathrm{ng} / \mathrm{ml}$ & 13 a 17 años $1,4-7,2 \mathrm{mg} / 24$ horas \\
\hline Ácido homovanílico & $0,8 \mathrm{mg} / 24$ horas & $10-15$ años 0-10 $\mu \mathrm{gg} / \mathrm{mg}$ creatinina \\
\hline Ácido vanilmandélico & $10,9 \mu \mathrm{g} / \mathrm{mg}$ creatinina & Menor \\
\hline
\end{tabular}

hallazgo se solicita valoración por Endocrinología. Los estudios hormonales apoyan el diagnóstico de feocromocitoma (tabla 2). Se realiza preparación prequirúrgica con prazocin y propranolol. Urología programa adrenalectomía laparoscópica, en la cual se encontró masa de $9 \times 6 \mathrm{~cm}$ adherida al hilio renal derecho, se realiza adrenalectomía más nefrectomía ipsilateral (figura 8). La patología confirma el diagnóstico de feocromocitoma.

Figura 8. Masa adrenal derecha. CASO 3

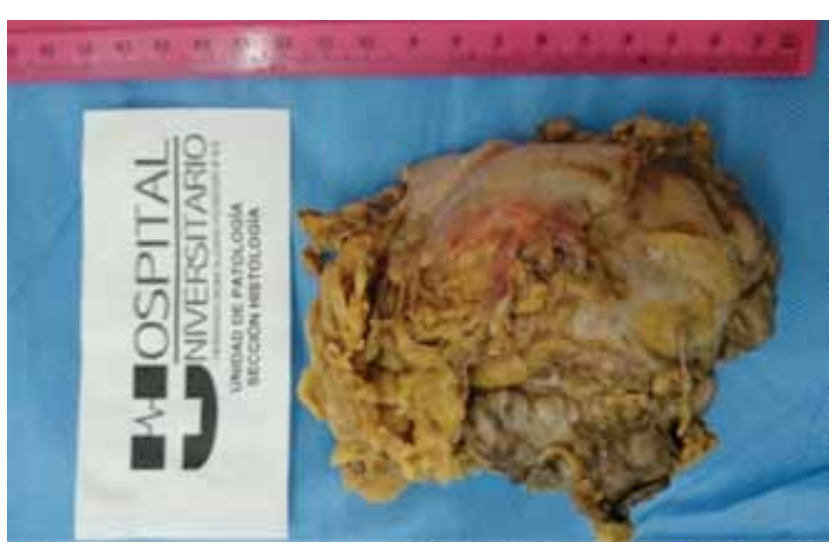

\section{Revision del tema}

El feocromocitoma es un tumor productor de catecolaminas de origen neuroectodérmico derivado de células cromafines $^{(3)}$. En 2004, la OMS define al feocromocitoma como un paraganglioma intraadrenal, mientras que los extraadrenales simpático o parasimpático se clasifican como paragangliomas $^{(4)}$. Se producen en 80 a $85 \%$ de los casos a partir de las células cromafines de la médula suprarrenal y 15 a $20 \%$ son extradrenales ${ }^{(5)}$. Estos se hallan en las cadenas simpáticas o en el órgano de Zuckerkandl; menos de $1 \%$ son intratorácicos ${ }^{(3)}$. Al- gunas localizaciones extradrenales son: intrapericárdicas, del tabique interauricular, próstata y vejiga ${ }^{(5)}$. La prevalencia de estos tumores es de 2 a 8 casos por millón de personas, con mayor incidencia en la tercera y cuarta década de la vida ${ }^{(6)}$. Los casos esporádicos se presentan entre los 40 y 50 años; los heredados, generalmente antes de los 40 $\operatorname{años}^{(7)}$. La incidencia estimada en población general es de $0,005 \%$ a $0,1 \%$ y de $0,1 \%$ a $0,2 \%$ en la población hipertensa adulta ${ }^{(8)}$.

\section{Síndromes genéticos asociados al feocromocitoma}

Nueve genes han sido asociados a feocromocitomas ${ }^{(9)}$. Muchos tienen penetrancia incompleta y/o expresión variable. La historia familiar positiva, un síndrome específico, múltiples tumores primarios, edad temprana al diagnóstico, malignidad o ubicación extradrenal, son todos factores que se deben considerar para la presencia de una mutación ${ }^{(10)}$.

\section{Síndrome de Von Hippel-Lindau (Vhl)}

Es un trastorno autosómico dominante con penetrancia del $97 \%$ a los 60 años, posee expresión variable inter e intrafamiliar ${ }^{(11)}$; con mutaciones puntuales del gen supresor de tumores VHL, lo que produce diferentes tumores: del sistema nervioso central, de retina, de células claras renales, tumores de islotes pancreáticos y feocromocitoma ${ }^{(12)}$. El $40 \%$ de los feocromocitomas familiares o bilaterales se asocian a mutaciones del gen $\mathrm{VHL}^{(13)}$. Este gen se localiza en el cromosoma 3 p25-26 e interviene en el ciclo celular, la estabilidad del ARNm y la regulación en la expresión de genes inducibles por hipoxia( ${ }^{(14)}$. Una función es la degradación por proteosomas del factor inducible por hipoxia 1 alfa (HIF-1 $\alpha$ ), el cual está implicado en la formación de vasos sanguíneos ${ }^{(15)}$. Los tumores asociados al síndrome VHL producen norepinefrina, pero no epinefrina, la edad media del diagnóstico es 28 años y la mitad son bilaterales o multicéntricos, pero rara vez malignos $(5 \%)^{(5,9)}$.

\section{Neurofibromatosis tipo 1}

La neurofibromatosis tipo 1 es un trastorno autosómico dominante que afecta a una de cada 2.500 a 3.000 personas en el mundo; es causada por mutaciones de inactivación en el gen NF1; un gen que se encuentra en el cromosoma 17 q11.2, el cual codifica para la neurofibromina, una GTPasa que regula de forma negativa el RAS e inhibe la vía de señalización MAPK, controlando el crecimiento y la diferenciación celular ${ }^{(13)}$. La predisposición a desarrollar tumores multisistémicos produ- 
ce un riesgo cuatro veces mayor, comparado con la población general, y del 0,1 al 5,7\% desarrollan feocromocitomas ${ }^{(16,17)}$. En el grupo de hipertensos pueden estar en 20 a $50 \%$ de los $\operatorname{casos}^{(18)}$ y en autopsias se encuentra en 3,3 a $13 \%{ }^{(19)}$. Dos estudios independientes ${ }^{(20,21)}$ han revelado que el gen de NF1 es el blanco más frecuente de mutaciones ( 41 y $42 \%$ ) en los feocromocitomas esporádicos ${ }^{(22)}$. El diagnóstico de la NF1 se realiza con criterios clínicos, los pacientes deben tener al menos dos de las siguientes características: seis o más manchas café con leche, dos o más neurofibromas cutáneos o un solo neurofibroma plexiforme; pecas inguinales o axilares; dos o más hamartomas del iris; glioma del nervio óptico; displasia de huesos largos; y un pariente de primer grado con NF1 ${ }^{(17)}$. La edad media al diagnóstico del feocromocitoma en estos pacientes es en promedio de 41 años ${ }^{(10)}$, son productores de epinefrina y norepinefrina, y cerca del $9,6 \%$ en la NF1 son bilaterales, $6 \%$ son extradrenales ${ }^{(13)}$ y 3 a $12 \%$ son malignos ${ }^{(15)}$.

\section{Neoplasia endocrina múltiple tipo 2 (MEN 2)}

El MEN 2 es un síndrome autosómico dominante caracterizado por la presencia de carcinoma medular de tiroides y feocromocitoma. Su incidencia es de 1:35.000 personas y se presenta en dos subtipos: MEN2A, que se caracteriza por carcinoma medular de tiroides (95\%), feocromocitoma (50\%) e hiperparatiroidismo primario (15\% a $30 \%)$; y MEN2B: carcinoma medular de tiroides y feocromocitoma en la mitad de los casos, hábito marfanoide y neuromas de mucosa ${ }^{(13)}$. Es producido por mutaciones en el protooncogén RET (10 q11.2). RET codifica un receptor tirosina cinasa que regula la proliferación y la apoptosis celular ${ }^{(5)}$. Las mutaciones se hallan en los codones 634, exón 11; y los codones 620, 618, 611, y 609; exón 10. La mutación del codón 634 está presente en el 90\% de los MEN2A que desarrollan feocromocitoma ${ }^{(5)}$. La mitad de los MEN2A y MEN2B tienen riesgo de desarrollar feocromocitoma, puede ser la forma de presentación en el 10 a 30\% de los casos, la edad promedio de presentación es de 36 años; y el 50 a $80 \%$ son sincrónicos o metacrónicos, multifocales o bilaterales. Casi nunca son extradrenales y menos de $5 \%$ son malignos ${ }^{(4)}$.

\section{Genética del feocromocitoma no sindromático}

Varias alteraciones genéticas están asociadas a predisposición de presentar feocromocitomas. La succinato deshidrogenasa (SDH) es una enzima heterotetramérica con subunidades (A, B, C y D). Este complejo se encuentra anclado a la pared interna de la mitocondria a través de SDHC y SDHD y tiene un papel clave en la producción de energía celular ${ }^{(10)}$. La pérdida de su función se asocia al desarrollo de feocromocitomas, principalmente por alteración de SDHD y SDHB. La alteración puede imitar a la hipoxia crónica y aumentar la actividad de HIF-1 $\alpha$ que conduce a proliferación celular ${ }^{(15)}$. La mutación en SDHD que se ubica en el cromosoma 11, es responsable del síndrome autosómico dominante de paragangliomas (PGL) tipo 1, se caracteriza por tumores familiares extradrenales simpáticos y parasimpáticos de cabeza y cuello, y de feocromocitomas benignos $^{(9)}$. El riesgo de desarrollar un PGL extradrenal es del $29 \%$ y se asocia a un riesgo de malignidad menor al $5 \%{ }^{(6)}$. El síndrome PGL 4 se produce por una mutación de inactivación en SDHB; está asociado con feocromocitomas extradrenales y un riesgo de malignidad en la mitad de los casos. Estas mutaciones son la causa más común de feocromocitomas malignos $^{(13)}$. La edad media al diagnóstico es de 29 a 37 años $^{(6)}$.

Otros genes que aumentan la susceptibilidad son: el gen KIF1B $\beta$, TMEM127 y MAX. Todos son genes supresores de tumor que, al perder su función, aumentan el riesgo para desarrollar neoplasias ${ }^{(11)}$. El solicitar pruebas genéticas depende de cada caso; pacientes menores de 45 años, tumores múltiples, extradrenales, malignos o la presencia de historia familiar, son indicaciones para realizarlas ${ }^{(5)}$.

\section{Feocromocitomas esporádicos}

La mayoría de los feocromocitomas son esporádicos; los pacientes son mayores y tienen una menor tasa de tumores múltiples que aquellos con enfermedad familiar. La tasa de mutaciones heredadas con historia familiar negativa es del 11 al $24 \%{ }^{(4,23,24)}$. Entre 340 pacientes, el $73 \%$ eran feocromocitomas esporádicos, el compromiso bilateral se presentó en $6 \%$, la edad media de presentación fue de 48 años y el 9\% tenían enfermedad maligna ${ }^{(25)}$. De forma peculiar, los feocromocitomas esporádicos poseen representación en los síndromes hereditarios ${ }^{(26,27)}$.

\section{Síntesis de catecolaminas y sus metabolitos}

El diagnóstico de feocromocitoma se basa en la evidencia bioquímica de la producción elevada de catecolaminas por el tumor $^{(4)}$. El proceso comienza con la conversión de la tirosina a 3,4-dihidroxifenilalanina (DOPA) por la enzima tirosina hidroxilasa. DOPA se convierte en dopamina que desde el citoplasma se almacena en vesículas de catecolaminas en las células cromafines de la médula adrenal, los nervios simpáticos y los paraganglios. La presencia de la enzima dopamina- $\beta$ hidroxilasa dentro de las vesículas es responsable de la conversión de dopamina en noradrenalina. La noradrenalina se convierte en adrenalina por la feniletanolamina-N-metiltransferasa que está presente exclusivamente dentro de la médula adrenal. La noradrenalina deriva de las neuronas noradrenérgicas y del sistema nervioso simpático. El metabolismo de las catecolaminas resulta en varios metabolitos. La desaminación neuronal de noradrenalina a 3,4-dihidroxifenilglicol (DHPG) se produce por la monoaminoxidasa (MAO) neuronal, también se metaboliza en los tejidos extraneuronales y adrenal donde se convierte en normetanefrina por la catecol-O-metiltransferasa (COMT). La adrenalina se metaboliza principalmente por la COMT a su metabolito 0-metilado ${ }^{(28)}$. 


\section{Diagnóstico de feocromocitoma}

El tamizaje se realiza a pacientes con incidentalomas adrenales, con predisposiciones genéticas y aquellos con manifestaciones sugestivas de feocromocitoma: cefalea paroxística, sudoración, taquicardia, palidez, náuseas, enrojecimiento, hipertensión, variabilidad inexplicable de la presión arterial, respuesta paradójica de la presión arterial a la anestesia, cirugía o medicamentos; e hipotensión ortostática en hiperten$\operatorname{sos}^{(4,29)}$. La medición de metanefrinas en plasma u orina son las pruebas que tienen mayor precisión diagnóstica ${ }^{(4,30)}$. Las mediciones de catecolaminas plasmáticas y urinarias son insuficientes, debido a que la secreción de catecolaminas es episódica o insignificante en pacientes asintomáticos. La precisión diagnóstica superior de las metanefrinas se atribuye a su continua producción y secreción a la circulación independiente a la de las catecolaminas ${ }^{(28)}$. Las metanefrina, libres en plasma tienen una sensibilidad del 96 al 99\% y una especificidad del 80 al 100\% (tabla 3) ${ }^{(28)}$. Aunque el ácido vanilmandélico urinario ofrece una alta especificidad, posee baja sensibilidad ${ }^{(30)}$. Resultados falsos positivos se deben tener en cuenta para la correcta interpretación de las pruebas, el aumento de la actividad simpática se puede presentar en relación con la postura, el ejercicio, la comida, el estrés, la hipoglucemia y el uso de algunos medicamentos ${ }^{(4)}$. Una muestra de sangre ideal para medir metanefrinas se debe tomar después de un descanso de 20 minutos, en posición supina, en un ambiente tranquilo; todo, importante para el análisis de la normetanefrina, el metabolito más sensible a la activación simpática ${ }^{(28)}$. Productos alimenticios como plátano y piña, frutos secos y cereales, contienen cantidades sustanciales de aminas biógenas que pueden producir resultados falsos positivos, particularmente si se va a medir 3 metoxitiramina ${ }^{(31)}$. Los fármacos pueden

Tabla 3. Sensibilidad y especificidad de las pruebas bioquímicas para la detección de feocromocitoma y paraganglioma

\begin{tabular}{l|c|c|c|c}
\hline \multicolumn{1}{c|}{$\begin{array}{c}\text { Prueba } \\
\text { bioquímica }\end{array}$} & Sensibilidad & \multicolumn{2}{l}{ Especificidad } & \\
\hline & NIÑOS & ADULTOS & NIÑOS & ADULTOS \\
\hline $\begin{array}{l}\text { Normetanefrina y } \\
\text { metanefrina en plasma }\end{array}$ & $100 \%$ & $99 \%$ & $94 \%$ & $89 \%$ \\
\hline $\begin{array}{l}\text { Norepinefrina y epinefrina } \\
\text { en plasma }\end{array}$ & $92 \%$ & $84 \%$ & $91 \%$ & $81 \%$ \\
\hline $\begin{array}{l}\text { Normetanefrina y } \\
\text { metanefrina urinaria }\end{array}$ & $100 \%$ & $97 \%$ & $95 \%$ & $69 \%$ \\
\hline $\begin{array}{l}\text { Norepinefrina y epinefrina } \\
\text { urinaria }\end{array}$ & $100 \%$ & $86 \%$ & $83 \%$ & $88 \%$ \\
\hline $\begin{array}{l}\text { Ácido vanilmandélico } \\
\text { urinario }\end{array}$ & - & $64 \%$ & - & $95 \%$ \\
\hline
\end{tabular}

Adaptado de The North American Neuroendocrine Tumor Society Consensus Guideline for the Diag nosis and Management of Neuroendocrine Tumors. Páncreas 2010. interferir de manera analítica o farmacodinámica con las mediciones de catecolaminas y sus metabolitos; por ejemplo, el acetaminofén puede afectar los ensayos de cromatografía de fase líquida (HPLC) de metanefrinas libres en plasma ${ }^{(32)}$. Numerosos productos aumentan las catecolaminas y sus metabolitos; dentro de estos figuran: la efedrina, las anfetaminas, la cocaína, la cafeína y la nicotina, que aumentan la liberación de noradrenalina y adrenalina ${ }^{(33)}$. Los medicamentos que inhiben la recaptación de serotonina y noradrenalina (por ejemplo: venlafaxina), y antidepresivos tricíclicos (ATC), pueden aumentar las concentraciones de noradrenalina y normetanefrina; al igual que los inhibidores de MAO que bloquean la conversión de noradrenalina y adrenalina a $\mathrm{DHPG}^{(28)}$. Los bloqueadores de canales de calcio dihidropiridínicos y los bloqueadores de receptores adrenérgicos $\alpha-1$ selectivos también pueden dar resultados falsos positivos, debido a la activación simpática refleja ${ }^{(30)}$.

La elevación en plasma de las metanefrinas más de 4 veces por encima del límite de referencia superior se asocia con una probabilidad del $100 \%$ de presentar un feocromocitoma ${ }^{(34)}$. Este resultado debe ser utilizado para determinar la necesidad de estudios de localización de tumores frente a investigaciones bioquímicas adicionales ${ }^{(4)}$. Pruebas bioquímicas adicionales son necesarias en resultados falsos positivos o por muestreos inadecuados. La prueba de supresión con clonidina combinada con mediciones de catecolaminas plasmáticas puede resultar útil(30).

\section{Estudio imaginológico del feocromocitoma}

La tomografía computarizada o la resonancia magnética se recomiendan para la localización inicial del tumor ${ }^{(35)}$. Los feocromocitomas tienen imágenes variables en la TAC, las lesiones pequeñas son homogéneas con densidad de tejidos blandos pero las masas grandes son a menudo heterogéneas, algunas quísticas, sólidas, con calcificaciones y áreas de necrosis o hemorragia $^{(36)}$. En la RM, los feocromocitomas han sido descritos como marcadamente hiperintensos en las imágenes T2; sin embargo, algunas series han mostrado que también pueden ser hipointensos ${ }^{(37)}$. En T1 son hipointensos y suelen mostrar realce después de administrar el contraste, no hay características radiológicas específicas para el diagnóstico de malignidad diferente a la invasión de estructuras locales o las metástasis a distancia ${ }^{(36)}$.

Las imágenes funcionales en feocromocitoma se pueden dividir en dos categorías: las específicas, que utilizan la síntesis de catecolaminas como metayodobencilguanidina (MIBG), y las inespecíficas que incluyen receptores de somatostatina (octreótide) y la 
fluoro-2-desoxi D glucosa (18F-FDG). Estos tipos de agentes se pueden visualizar con gammagrafía convencional o por tomografía por emisión de positrones (PET) ${ }^{(38)}$. La MIBG se ha utilizado para el diagnóstico, debido a su parecido con la norepinefrina y su absorción por el trasportador de membrana de catecolaminas; este estudio posee una sensibilidad de 77 a $90 \%$ y una especificidad de 95 a $100 \%{ }^{(39)}$. Aunque el octreoscan tiene un rendimiento inferior a la MIBG, es de mayor rendimiento en lesiones metastásicas ${ }^{(38)}$. La PET con 18F-FDG refleja la absorción excesiva de glucosa principalmente a través de GLUT-1 en tumores metabólicamente hiperactivos y posee mayor rendimiento comparada con MIGB especialmente en tumores malignos ${ }^{(35)}$. La literatura recomienda una imagen funcional en todos los feocromocitomas, excepto en los casos no familiares con un diagnóstico bioquímico claro y una masa adrenal unilateral ${ }^{(39)}$. Sin embargo, aquellos con fenotipo bioquímico noradrenérgico pueden tener una lesión adrenal, extradrenal, o ambas y también necesitan imagen funcional ${ }^{(39)}$.

\section{Diagnóstico de feocromocitoma maligno}

El diagnóstico clínico e histopatológico de malignidad es difícil y controvertido, algunas características histológicas asociadas a malignidad son: tamaño tumoral mayor de $5 \mathrm{~cm}$, necrosis tumoral, Ki67 mayor de 4\% y ausencia de PS100. Los métodos más conocidos de puntuación histopatológica para predecir metástasis poseen pobre concordancia ${ }^{(40)}$. El diagnóstico se basa en documentar la invasión de los órganos adyacentes o la presencia de metástasis ${ }^{(3,13)}$. Los sitios más frecuentes de metástasis son: el hígado, el pulmón y las vértebras, el cráneo o las costillas ${ }^{(3)}$

\section{Tratamiento del feocromocitoma}

Los riesgos intraoperatorios deben minimizarse con un tratamiento preoperatorio para bloquear los efectos de las catecolaminas durante 10 a 14 días antes de cirugía. Un adecuado bloqueo alfa reduce las complicaciones a menos del $3 \%{ }^{(41)}$. La fenoxibenzamina, un bloqueador alfa adrenérgico, es el medicamento más recomendado en la literatura para el control preoperatorio de la presión arterial ${ }^{(42)}$.En Colombia, este medicamento no se encuentra disponible, por lo que utilizamos bloqueadores competitivos de los receptores alfa 1 (doxazosin, terazosin y prazocin) y bloqueadores de los canales de calcio. Bloqueadores beta se usan para prevenir taquiarritmias o angina; el más recomendado es el propranolol, un bloqueador no selectivo con vida media mayor de 4 horas. Generalmente se requieren de 80 a $120 \mathrm{mg} /$ día, aunque en feocromocitomas secretores de epinefrina se pueden necesitar dosis de hasta $480 \mathrm{mg}$ al día ${ }^{(43)}$. La pérdida de la vasodilatación mediada por el receptor beta en un paciente sin oposición a la vasoconstricción inducida por catecolaminas, puede dar lugar a aumentos peligrosos de la presión arterial, por lo que es esencial el adecuado bloqueo alfa antes del inicio del bloqueo beta. ${ }^{4}$ La contracción de volumen asociada con la vasoconstricción crónica es vista en pacientes con feocromocitoma, por ello se recomienda la expansión de volumen preoperatorio mediante infusión de solución salina o el aumento en la ingesta de agua para reducir la hipotensión posoperatoria $^{(42)}$. La hipertensión y los cambios repentinos de tensión arterial durante la cirugía se pueden manejar de forma agresiva con el uso de medicamentos intravenosos potentes, de acción corta, como nitroprusiato de sodio y nitroglicerina(44).

La cirugía es el tratamiento primario del feocromocitoma y la técnica laparoscópica es la primera opción para la resección de tumores adrenales y extradrenales. Esta intervención reduce la morbilidad posoperatoria, la estancia hospitalaria y los gastos en comparación con la técnica transabdominal convencional; sin embargo, los enfoques quirúrgicos abiertos podrían seguir siendo necesarios en casos de enfermedad localmente invasiva o maligna ${ }^{(45)}$. Debido a la alta incidencia de enfermedad adrenal bilateral en el feocromocitoma hereditario, la adrenalectomía parcial se recomienda para evitar la morbilidad asociada con el remplazo médico adrenal permanente ${ }^{(4)}$.

\section{Manejo de la enfermedad avanzada}

La cirugía paliativa se realiza para liberar la presión del tumor sobre los tejidos circundantes o para disminuir la masa tumoral. La disminución de la carga tumoral puede conducir a una disminución significativa en la secreción de catecolaminas y facilita la posterior radio o quimioterapia, a pesar de que no está probada una mayor supervivencia luego de citorreducción quirúrgica ${ }^{(45)}$. En algunas lesiones metastásicas, la ablación por radiofrecuencia y crioablación son opciones actuales. El uso de yodo 131 en MIBG se utiliza para pacientes en los que la gammagrafía MIBG es positiva ${ }^{(4,45)}$. En pacientes con tumores de rápido crecimiento y gammagrafía MIBG negativa, la quimioterapia es una opción de tratamiento. Estos esquemas pueden proporcionar regresión del tumor y alivio de síntomas en la mitad de los casos, pero las respuestas son de corta duración ${ }^{(4,46)}$

\section{Discusión}

Los tres casos ilustrados en esta revisión son los presentados en el último año en nuestro hospital, de una patología rara que posee diferentes cuadros clínicos. Aunque no se encuentra una clara predisposición por sexo, los tres pacientes son hombres, con un amplio rango de edad al momento del diagnóstico y con lesiones adrenales que por tamaño tenían indicación de manejo quirúrgico. El paciente del caso 1 es llevado a biopsia y el paciente del caso 2 es llevado a cirugía sin estudios bioquímicos previos, exponiéndolos a los riesgos relacionados a descargas catecolaminérgicas o a inadecuada preparación quirúrgica. De ello, la importancia en la valoración por Endocrinología de toda lesión adrenal para realizar 
el adecuado estudio bioquímico previo a la toma de conductas intervencionistas; como se realizó en el caso número tres. El manejo preoperatorio se realiza con bloqueo adrenérgico alfa, usando fenoxibenzamina, la cual no se encuentra disponible en nuestra región, por lo que usamos alternativas como el prazocin, un fármaco disponible, económico y efectivo, previo al bloqueo betaadrenérgico.

Actualmente no existe una forma efectiva para la diferenciación prequirúrgica entre feocromocitoma benigno y maligno; el diagnóstico clínico e histopatológico de malignidad es difícil y

\section{Referencias}

1. De Wailly P, Oragano L, Radé F, Malignant pheochromocytoma: new malignancy criteria. Langenbecks Arch Surg 2012; 397:239-46.

2. Neumann HPH, Bausch B, Mcwhinney SR, Bender BU, Gimm O, Franke G, Schipper J, Klisch J, Altehoefer C, Zerres K, Januszewicz A, Eng C, Germ-line mutations in non syndromic pheochromocytoma. N Engl J Med 2002; 346: 1459-1466.

3. Mittendorf E, Evans D, Lee J, Perrier N, Pheochromocytoma: Advances in Genetics, Diagnosis, Localization, and Treatment. Hematol Oncol Clin N Am 21 (2007) 509-525.

4. Neumann HPH, Bausch B, Mcwhinney SR, Bender BU, Gimm O, Franke G, Schipper J, Klisch J, Altehoefer C, Zerres K, Januszewicz A, Eng C, Germ-line mutations in non syndromic pheochromocytoma. N Engl J Med 2002; 346: 1459-1466.

5. Chen H , Sippel R, O'Dorisio M, Vinik A, Lloyd R, Karel, The North American Neuroendocrine Tumor Society Consensus Guideline for the Diagnosis and Management of Neuroendocrine Tumors. Pancreas 2010; 39: 775-783.

6. Lefebvre M, Foulkes W.D., Pheochromocytoma and paraganglioma syndromes: genetics and management update. Curr Oncol, 2014; 21: 8-17.

7. Fishbein L, Nathanson K, Pheochromocytoma and paraganglioma: understanding the complexities of the genetic background. Cancer Genet 205, 2012, 1-11.

8. Gimenez-Roqueplo F, Genetics of paragangliomas and pheochromocytomas [French]. Med Sci (Paris) 2012; 28:625-32.

9. Jafri M, Maher E, The genetics of pheochromocytoma: using clinical features to guide genetic testing. Eur J Endocrinol 2012:166,151-158.

10. Krishnappa R, Chikaraddi SB, Arun HN, Deshmane V, Pheochromocytoma in Indian patients: A retrospective study. Indian J Cancer 2012; 49:188-93.

11. Maher E, Neumann H, Richard S, Von Hippel-Lindau disease: A clinical and scientific review. Eur J Hum Genet (2011) 19, 617-623.

12. Opocher G, Schiavi, Genetics of pheochromocytomas and paragangliomas. Best Pract Res Clin Endocrinol Metab 24 (2010) 943-956.

13. Vicha A, Musil Z, Pacak K, Genetics of pheochromocytoma and paraganglioma syndromes: new advances and future treatment options. Curr Opin Endocrinol Diabetes Obes 2013, 20:186-191.

14. Kolačkov K, Tupikowski K, Bednarek-Tupikowska G, Genetic Aspects of Pheochromocytoma. Adv Clin Exp Med 2012, 21, 6, 821-829.

15. Kaelin Jr. WG. Molecular basis of the VHL hereditary cancer syndrome. Nat Rev Cancer 2002; 2(9): 673-682.

16. Mazzaglia P., Hereditary Pheochromocytoma and Paraganglioma. J Surg Oncol 2012; 106:580-585.

17. Knudson Jr. AG, Mutation and cancer: statistical study of retinoblastoma. Proc Natl Acad Sci USA 1971; 68(4): 820-823.

18. Hirbe A, Gutmann D, Neurofibromatosis type 1: a multidisciplinary approach to care. Lancet Neurol 2014; 13: 834-43.

19. Burnichon $\mathrm{N}$, mutations cause hereditary and sporadic pheochromocytoma and paraganglioma. Clin Cancer Res 2012:18, 2828-2837.

20. Welander J, Soderkvist P, Gimm O, Genetics and clinical characteristics of hereditary pheochromocytomas and paragangliomas. Endocr Relat Cancer. 2011: $1 ; 18,253-276$.

21. Jimerez C, Cote G, Arnold A, Gagel RF. Review: should patients with apparently sporadic pheochromocytomas or paragangliomas be screened for hereditary syndromes? J Clin Endocrinol Metab 2006; 91:2851-8.

22. Walther MM, Herring J, Enquist E, Keiser HR \& Linehan WM. Von Recklinghausen's disease and pheochromocytomas. J Urol 1999; 162(5): 15821586.

23. Welander J, Soderkvist P, Gimm O. The NF1 gene: a frequent mutational target in sporadic pheochromocytomas and beyond. Endocr Relat Cancer 2013:20, 13-17.

24. Lenders J, Duh Q Eisenhofer G, Gimenez-Roqueplo A, Grebe S, Murad M “et al", Pheochromocytoma and Paraganglioma: An Endocrine Society Clinical Practice Guideline. J Clin Endocrinol Metab, 2014 : 99(6):1915-1942. controvertido. Si se tiene en cuenta la definición de la Organización Mundial de la Salud, en donde se establece como tumor maligno el que presenta invasión local o el que se asocia a lesiones metástasicas a distancia; toman importancia definitiva los estudios funcionales con MIBG y el PET o la fusión PET - TAC; métodos de referencia para descartar compromiso metastásico.

La resección laparoscópica fue usada en los tres casos sin complicaciones y buena respuesta final. En nuestro medio mantenemos la limitante de realizar estudios genéticos para confirmar las mutaciones descritas en la literatura mundial.

25. Waldmann J, Langer P, Habbe N, Fendrich V, Ramaswamy A, Rothmund M, Bartsch DK, Slater EP , Mutations and polymorphisms in the SDHB, SDHD VHL, and RET genes in sporadic and familial pheochromocytomas. Endocrine 2009 : 35 347-355.

26. Mannelli M, Ercolino T, Giache V, Simi L, Cirami C, Parenti G, Genetic screening for pheochromocytoma: should SDHC gene analysis be included? J Med Genet 2007: 44 586-587.

27. Dahia PL, Ross KN, Wright ME, Hayashida CY, Santagata S, Barontini M, Kung AL, Sanso G, Powers JF, Tischler, A HIF1 alpha regulatory loop links hypoxia and mitochondrial signals in pheochromocytomas. PLoS Genet 2005: 1 , 72-80.

28. De Jong WH, de Vries EG, Kema IP. Current status and future developments of LC-MS/MS in clinical chemistry for quantification of biogenic amines. Clin Biochem 2011: 44 95-103.

29. EisenhoferS G, Screening for Pheochromocytomas and Paragangliomas Curr Hypertens Rep, 2012, 14:130-137.

30. Minnelli M, Lenders I , Pacak K, Parenti G, Eisenhofer G , Subclinical phaeochromocytoma. Best Pract Res Clin Endocrinol Metab 26, 2012: 507-515.

31. Van Berkel A, Lenders J, Timmers H, Biochemical diagnosis of phaeochromocytoma and paraganglioma. Eur J Endocrinol (2014) 170, R109-R119.

32. De Jong WH, Eisenhofer G, Post WJ, Muskiet FA, and de Vries EG, Kema IP. Dietary influences on plasma and urinary metanephrines: implications for diagnosis of catecholamine-producing tumors. J Clin Endocrinol Metab 200994 2841-2849.

33. Davidson FD. Paracetamol-associated interference in an HPLC-ECD assay for urinary free metadrenalines and catecholamines. Ann Clin Biochem 2004: 41 316-320.

34. Eisenhofer G, Goldstein DS, Walther MM, Friberg P, Lenders JW, Keiser HR , Pacak K. Biochemical diagnosis of pheochromocytoma: how to distinguish true- from false-positive test results. J Clin Endocrinol Metab 2003: 88 2656-2666.

35. Lumachi F, Tregnaghi A, Zucchetta P, Sensitivity and positive predictive value of CT, MRI and 123I-MIBG scintigraphy in localizing pheochromocytomas: a prospective study. Nucl Med Commun. 2006: 27(7):583Y587.

36. Blanchet E, Martucci V, Pacak K, Pheochromocytoma and paraganglioma: current functional and future molecular imaging. Front Oncol.2012:1,58

37. Song J, Mayo-Smith W, Current Status of Imaging for Adrenal Gland Tumors. Surg Oncol Clin N Am 23, 2014: 847-861

38. Jacques AE, Sahdev A, Sandrasagara M, Adrenal phaeochromocytoma: correlation of MRI appearances with histology and function. Eur Radiol 2008; 18:2885-92.

39. Khan S,Win Z, Szyszko T, Lloyd C, Dunn J, Alavi A, AL-Nahhas A, PET Imaging of Pheochromocytoma. PET Clin 2, 2008: 341-349.

40. Baudin E, Habra M, Deschamps F, Cote G, Dumont F, Cabanillas M "et al", Treatment of malignant pheochromocytoma and paraganglioma. Eur J Endocrinol, 2014: 171, 111-122.

41. Havekes B, King K, Lai E, Romijn J, Corssmit E, Pacak K ,New imaging approaches to phaeochromocytomas and paragangliomas. Clin Endocrinol. 2010: 72, 137-145.

42. Goldstein RE, O'Neill JA Jr, Holcomb GW, Clinical experience over 48 years with pheochromocytoma. Ann Surg. 1999; 229(6):755-764.

43. Zuber S, Kantorovich V, Pacak K, Hypertension in Pheochromocytoma: Characteristics and Treatment. Endocrinol Metab Clin N Am 40 (2011) 295-311.

44. Pacak K. Preoperative management of the pheochromocytoma patient. J Clin Endocrinol Metab. 2007; 92(11):4069-4079.

45. Domi R, Laho H. Management of pheochromocytoma: Old ideas and new drugs. Niger J Clin Pract 2012; 15:253-257.

46. Ayala-Ramirez M, Feng L, Johnson MM, Ejaz S, Habra MA, Rich T, Busaidy $\mathrm{N}$, Cote GJ, "et al": Clinical risk factors for malignancy and overall survival in patients with pheochromocytomas and sympathetic paragangliomas: primary tumor size and primary tumor location as prognostic indicators. J Clin Endocrinol Metab 2011: 96, 717-725. 